\title{
Radiological Diagnoses in the Context of Emigration: Infectious diseases
}

\section{Radiologische Diagnosen im Migrationskontext: Infektionskrankheiten}

Authors

Marija Stojkovic ${ }^{1}$, Jan Müller ${ }^{2}$, Thomas Junghanss ${ }^{1}$, Tim Frederik Weber ${ }^{2}$

Affiliation

1 Department of Clinical Tropical Medicine, University Hospital Heidelberg, Germany

2 Department of Diagnostic and Interventional Radiology, University Hospital Heidelberg, Germany

Key words

abdomen, brain, thorax, diagnostic radiology, infection, parasites

received 19.03.2017

accepted 19.09.2017

Bibliography

DOI https://doi.org/10.1055/s-0043-120764

Published online: 28.12.2017

Fortschr Röntgenstr 2018; 190: 121-133

(c) Georg Thieme Verlag KG, Stuttgart · New York

ISSN 1438-9029

Correspondence

PD Dr. Tim Frederik Weber

Diagnostic and Interventional Radiology, University Hospital Heidelberg, INF 410, 69120 Heidelberg

Tel.: ++ 49/62 21/5664 18

tim.weber@med.uni-heidelberg.de

\section{ABSTRACT}

Background Globalization and emigration impact on the spectrum of diseases challenging health care systems. Medical practitioners have to particularly prepare for infectious diseases.

Methods The database of a health care center specialized on tropical medicine was screened for patients with history of migration and one of the following diagnoses: Cystic echinococcosis, tuberculosis, schistosomiasis, visceral leishmaniosis, and neurocysticercosis. Representative casuistics were prepared from select case histories. Radiological pertinent knowledge was compiled based on literature search.

Results A small selection of frequently imported infectious diseases covers a considerable fraction of health care problems associated with migration. For cystic echinococcosis, schistosomiasis, and neurocysticercosis imaging is the most relevant diagnostic procedure defining also disease stages.
Tuberculosis and visceral leishmaniosis are important differentials for malignant diseases.

Conclusion Imaging plays a meaningful role in diagnosis, treatment stratification, and follow-up of imported infectious diseases. Radiological skills concerning these diseases are important for providing health care for patients in context of migration.

\section{Key Points}

- Imaging plays a meaningful role in multidisciplinary care for imported infectious diseases.

- A small selection covers a considerable fraction of infectious diseases expected in context of migration.

\section{Citation Format}

- Stojkovic M, Müller J, Junghanss T et al. Radiological Diagnoses in the Context of Emigration: Infectious diseases. Fortschr Röntgenstr 2018; 190: 121-133

\section{ZUSAMMENFASSUNG}

Hintergrund Globalisierung und Migration beeinflussen das Spektrum an Erkrankungen, mit dem die hiesigen medizinischen Versorgungseinrichtungen konfrontiert werden. Die Ärzteschaft muss sich insbesondere auf Infektionskrankheiten einstellen, die in unseren Breiten nie vorkamen oder nicht mehr vorkommen.

Methode Die Datenbank eines tropenmedizinischen Zentrums wurde auf Patienten mit Migrationshintergrund und Diagnose einer der folgenden Infektionskrankheiten durchsucht: Zystische Echinokokkose, Tuberkulose, Schistosomiasis, viszerale Leishmaniose und Neurozystizerkose. Aus ausgewählten Krankengeschichten wurden repräsentative Kasuistiken erstellt. Radiologisch bedeutsame Inhalte wurden auf Grundlage einer Literaturrecherche zusammengefasst.

Ergebnisse Bereits eine kleine Auswahl häufig importierter Krankheiten deckt einen beträchtlichen Anteil migrationsassoziierter Gesundheitsprobleme ab. Bei der zystischen Echinokokkose, der Schistosomiasis und der Neurozystizerkose ist die Bildgebung das diagnostisch entscheidende Verfahren. Mithilfe der Bildgebung wird bei diesen Erkrankungen auch das Therapie-bestimmende Staging durchgeführt. Tuberkulose und viszerale Leishmaniose sind wichtige Differentialdiagnosen zu malignen Erkrankungen. 
Schlussfolgerungen Die Radiologie spielt eine bedeutsame Rolle bei Diagnosestellung, Therapiestratifikation und Behandlungserfolgskontrolle von importierten Infektionskrankheiten.
Radiologische Kenntnisse zu diesen Erkrankungen sind wichtig für die medizinische Versorgung von Patienten im Migrationskontext.

\section{Introduction}

What is the effect of increasing globalization on the practice of medicine? We have to adapt to a new geographical and frequency distribution of diseases [1]. Travelers from wealthy countries - tourists and business people - differ in principle from emigrants who were born and raised in poor regions of the world. In the former, infectious diseases are usually present in their early forms, often detectable only using extensive laboratory procedures, whereas the latter exhibit "mature" stages of disease, already observable in a clinical and/or radiological examination. Often these diseases are radiological incidental findings. One example is schistosomiasis, unknowingly acquired by tourists when swimming in bodies of waters, such as Lake Victoria, which in mildly-infected persons is only discovered via indirect detection methods. This same infection in a teenager who grew up along such a lake might first be noticed by a radiologist who finds liver fibrosis and signs of portal hypertension.

Germany is an immigration country for workers, trainees and refugees. The number of foreign-born residents living in Germany is estimated to be 9.1 million persons. In 2011 alone the number of intercontinental air travelers exceeded the one billion mark [2]. This means that the medical profession has to adapt to diseases that have never occurred in our region or are no longer present, but which represent substantial morbidity and causes of mortality worldwide. In 2015, 955000 people emigrated to Germany, of whom 850000 were refugees. The majority of refugees in Europe currently come from Syria, Afghanistan, Eritrea, Somalia, Nigeria, Sudan and the Balkans. Their disease spectrum is quite different from that of our native population [3].

Radiologists can contribute to the detection of emigrationassociated diseases. A small spectrum of frequently imported diseases covers a considerable proportion of the health problems of immigrants which are presented here based on a case series resulting from our interdisciplinary collaboration at the Heidelberg University Hospital.

\section{Cystic Echinococcosis}

Cystic echinococcosis (CE) is diagnosed radiologically based on specific image characteristics of the cyst wall and matrix. Serology plays only a secondary role, and in a very high number of patients can provide a false negative result. This is observed especially in young cysts in WHO stage CE1 with a still-intact endocyst and absent antigen contact as well as in the case of old cysts in WHO stages CE 4 and CE 5. Therapy depends on the stage, quantity and location of the cysts. Treatment modalities include: watch and wait, surgical and drug therapy with benzimidazoles (mainly albendazole) and percutaneous procedures. However, percutaneous therapy involving instillation of protoscolic substances is increasingly viewed critically due to the risk of toxic cholangitis.
The human form of CE is caused by infection by the larvae of the dog tapeworm (Echinococcus granulosus) and is found worldwide. Primary areas of infection are the Mediterranean region, the Near and Middle East, North and East Africa, Tibet and China as well as the Andean region of Latin America. Incubation time can take years due to the slow growth rate of the cysts, most of which are found in the liver (70\%) or lung (20\%). The majority of patients are symptom-free. Symptoms arise when cysts reach a certain size or when cyst-related complications occur (cystobiliary and cystobronchial fistula, compression effects on organs and vessels, cyst rupture, cyst infection) [4].

Sonography is the radiological procedure of choice to identify and assess the activity of CE [4]. MRI is considered to have the same value when using heavily $\mathrm{T} 2$-weighted sequences except for young cysts [5]. CT is not a reliable means of assessing CE [5]. MRI or CT are indicated for subdiaphragmatic, disseminated or extra-abdominal disease, complicated cysts, and when planning surgical intervention [4]. Alveolar echinococcosis (AE) should be distinguished from CE, as it presents a separate disease with respect to epidemiology, radiology and prognosis.

Depending on the stage of the disease, pathognomonic CE signs allow delineation compared to cystic lesions of other etiologies. The WHO stage classification distinguishes among active, transitional and inactive echinococcal cysts [6]. - Fig. 1 demonstrates the sonographic features of the cyst stages.

Since they are univesicular cysts, young echinococcal cysts are sometimes indistinguishable from dysontogenetic cysts (CL, cystic lesion) Sonographic evidence of lamination of the cyst wall is confirmation of CE1. This describes the recognizable stratification of the cyst wall, which consists of the pericyst developed by the host and the parasitic endocyst. Absence of lamination does not rule out $\mathrm{CE}$. The sonographic test for hydatid sand, which can be detectable in active cysts, requires the cyst to be examined during a repositioning test in which the whirling hydatid sand shows a "snow storm" sign. Hydatid sand is made up of brood capsules separated from the germinative membrane of the endocyst.

The other cyst stages are determined by the characterization of the cyst matrix. CE2 cysts are multivesicular, in which the mother cyst is filled with daughter cysts, so that a so-called rosette-sign is recognizable in the cyst interior. Detachment of the endocyst from the pericyst gives rise to a pathognomonic garland-like structure (water lily sign) formed in univesicular CE1 cysts which defines the CE3a cyst stage.

Involution of cysts is characterized by the consolidation of the cyst content. The detection of daughter cysts in a solid cyst matrix is indicative of stage CE3b. Complete consolidation of the cyst characterizes the inactive CE4 stage. Detached endocysts or walls of daughter cysts can remain detectable as canalicular structures within the cyst matrix. Stage CE5 describes calcified inactive cysts. However, calcifications can appear at all cyst stages [7]. 

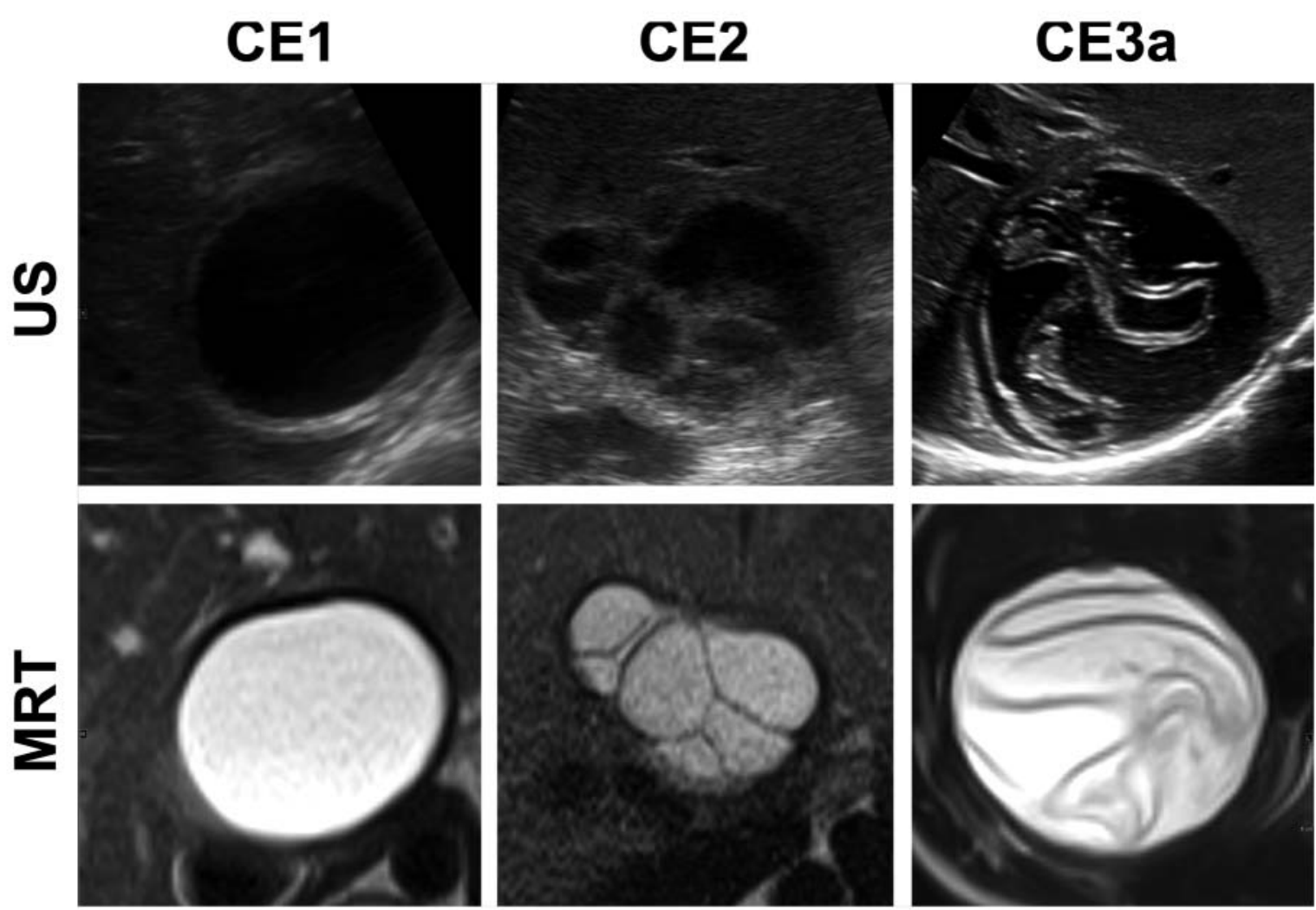

CE3b

CE4

CE5
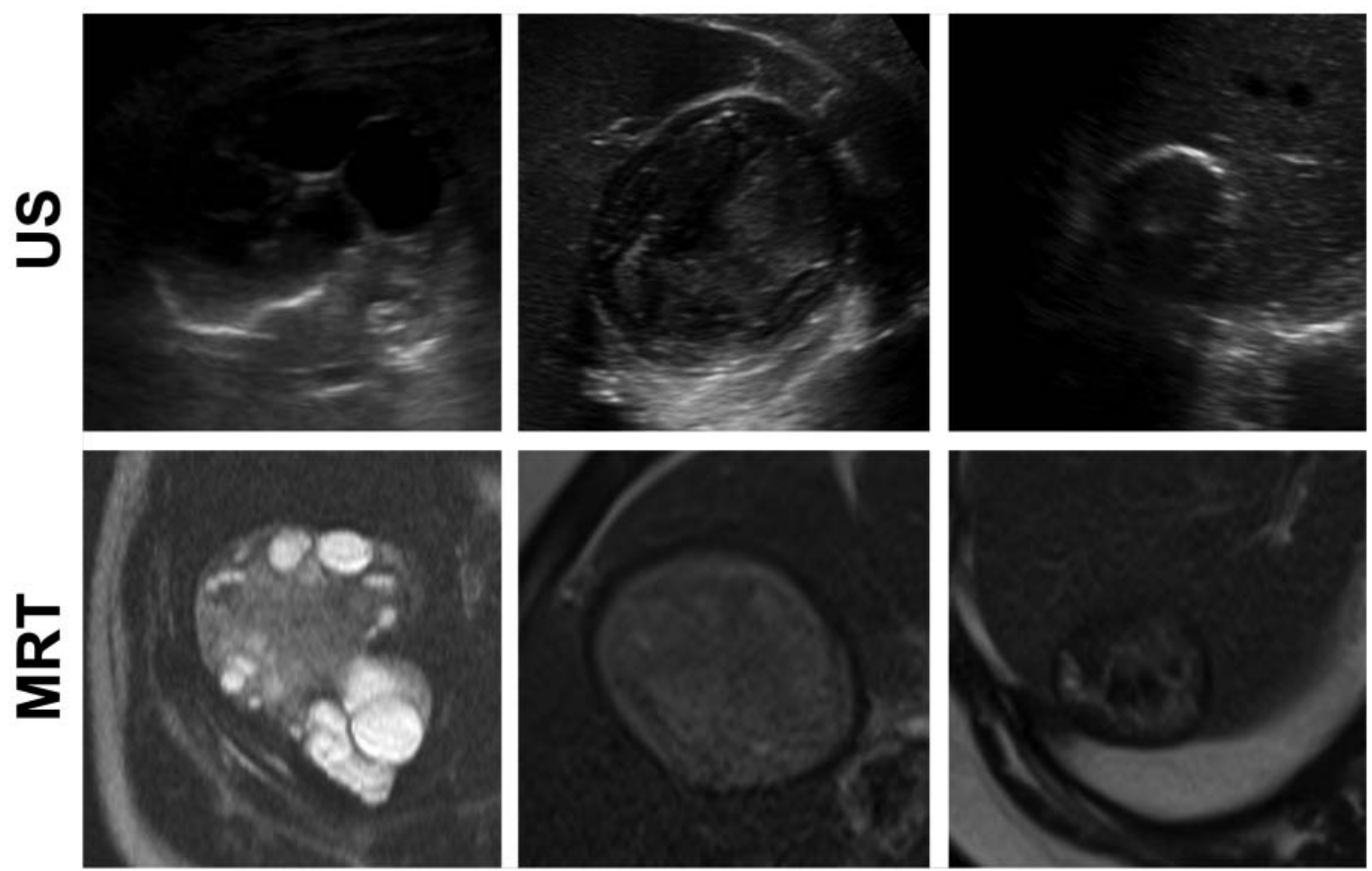

- Fig. 1 WHO classification for staging of cystic echinococcosis with characteristic imaging appearance in ultrasound (US) and MRI (MRT). 


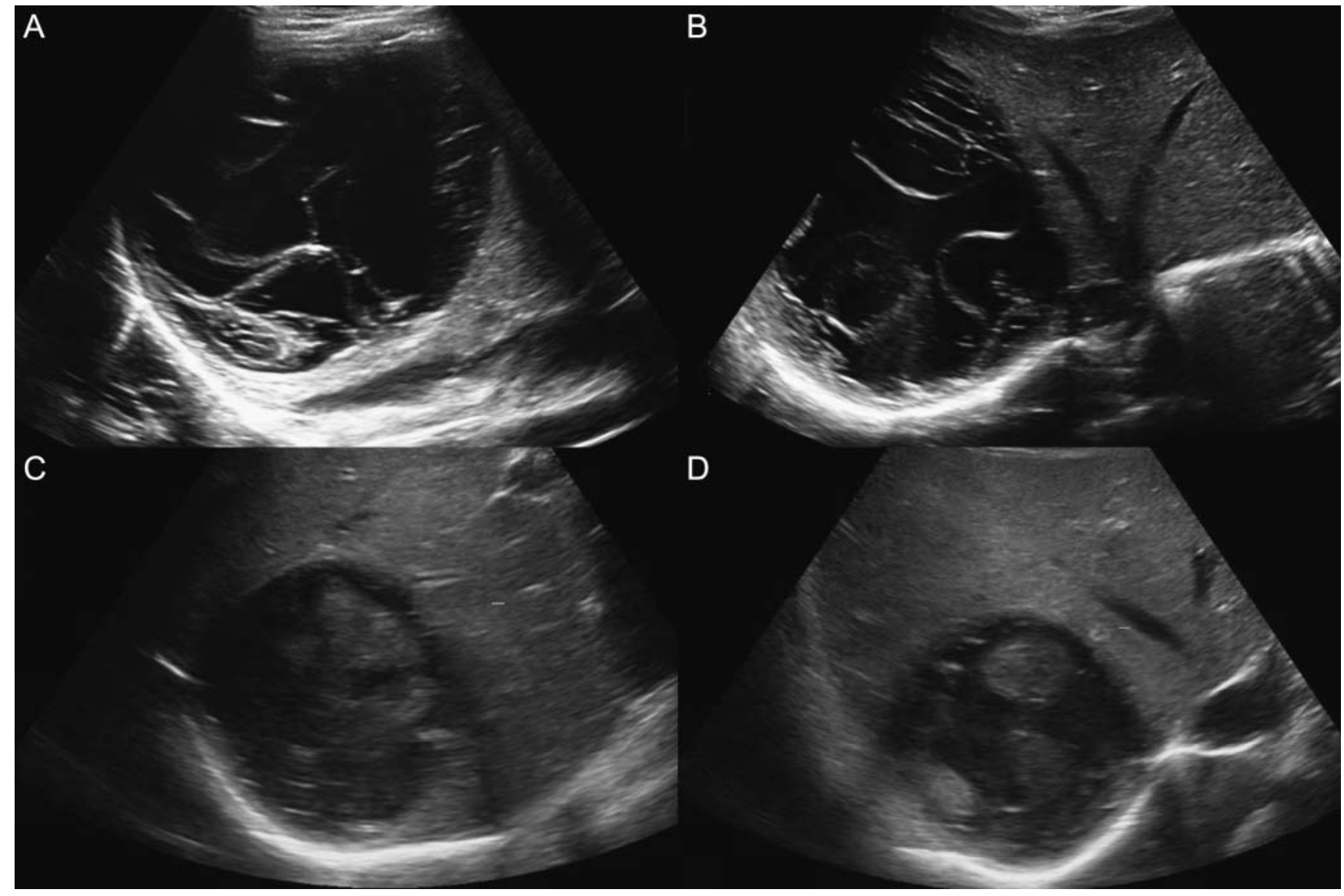

- Fig. 2 Patient with cystic echinococcosis of the lung. Contrast-enhanced CT shows a cystic mass in the right lung (star in A). In the right pulmonary apex and in the right lower lobe additional cystic masses with intralesional gas can be found (arrow heads in $\mathbf{B}$ and $\mathbf{C}$ ). These cysts are partially collapsed after rupture with endobronchial fistulization.

The differential diagnoses of CE depend on the cyst stage. CE1 and CE2 cysts must be differentiated from simple and complex dysontogenetic cysts. Cysts with solid components (CE3b and CE4) have to be distinguished from neoplasia. Indication of the presence of CE in this case is absent vascularization in contrast-enhanced examinations.

\section{Case History: Incidental Finding of CE of the Liver in a Pregnant Woman}

A 23-year-old female Syrian patient presented in a hospital because of epigastric pains. Sonography revealed a $12 \mathrm{~cm}$ univesicular cyst in the right lobe of the liver. Echinococcal serology was negative, therefore puncture and aspiration of $700 \mathrm{ml}$ water-clear liquid was performed. The puncture specimen exhibited microscopic presence of protoscolices resulting in post-puncture diagnosis of CE. Early pregnancy was determined post-puncture. Due to the puncture sample, an external decision was made to treat with albendazole. After our Center was contacted, albendazole was discontinued with a follow-up ( $\triangleright$ Fig. 2).
Abdominal ultrasound at this juncture revealed a univesicular cyst with water lily signs (CE3a). Control sonography 8 months later revealed increasing consolidation of the cyst. Since consolidation and related inactivation of the cyst was incomplete, birth by $\mathrm{C}$-section was chosen due to the risk of rupture.

\section{Case History: CE of the Lung}

Seven-year-old male patient from Syria. He presented to the pediatrician because of chronic cough. Chest X-ray revealed a mass in right upper lobe which was confirmed using CT. ( $\triangleright$ Fig. 3)

Contrast-enhanced CT showed a fluid-filled, sharply delineated cyst in the right upper lobe with displacing growth. Additional cystic lesions with gas beads were shown in the right apex and right lower lobe. Radiological diagnosis of CE of the lung was made. In addition to an intact-appearing cyst, two cysts complicated by cystobronchial fistulas were observed. Echinococcal serology was positive. Endocystectomy was performed.

Development of complications with rupture or cystobronchial fistulas is very common in pulmonary echinococcal cysts with a frequency of up to $90 \%$ [8, 9]. Epiphenomena often appear superimposed above radiological signs of CE. The presence of gas in a cystic mass, either as an isolated gas bubble, as an air-fluid level or crescent-shaped rim of air is indicative of CE $[9,10]$. 

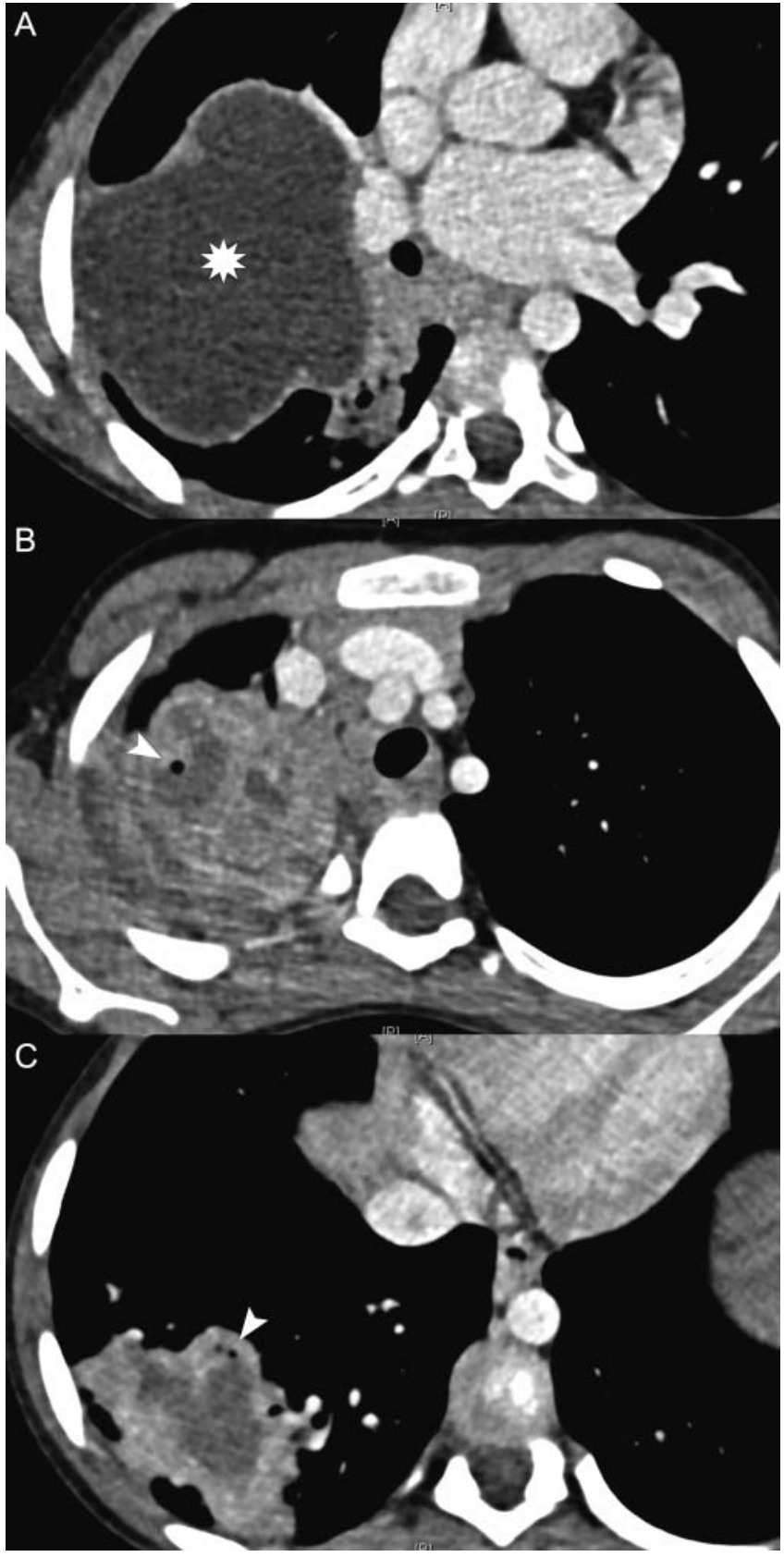

- Fig. 3 Patient with cystic echinococcosis of the lung. Contrastenhanced $\mathrm{CT}$ shows a cystic mass in the right lung (star in $\mathbf{A}$ ). In the right pulmonary apex and in the right lower lobe additional cystic masses with intralesional gas can be found (arrow heads in B and C). These cysts are partially collapsed after rupture with endobronchial fistulization.

\section{KEY POINTS}

- The liver and lungs are the most common manifestation sites of CE.

- CE is primarily diagnosed using imaging, since serology can provide false negative results in a high percentage of patients.
- Imaging procedures provide the stage classification relevant for therapeutic decisions.

- Pulmonary CE often presents with cystic complications.

\section{Tuberculosis}

In Germany, tuberculosis (TB) infection is generally a pulmonary or extrapulmonary postprimary reactivation of primary tuberculosis acquired in a patient's country of origin with a high incidence of TB. Reactivation usually takes place in the lung. There are usually no diagnostic difficulties with respect to its characteristic radiological presentation. Clinical delay in establishing a diagnosis is usually in cases of atypical (pulmonary) presentation, that is, no cavitation and no preferential location in the upper lobes, such as is observed among immunosuppressed or HIV patients and those with extrapulmonary postprimary TB. Extrapulmonary postprimary TB should be kept in mind when making a differential diagnosis for patients with suspected tumors from high-prevalence countries, where the likelihood of a malignancy compared to TB is reversed in comparison to our native population, especially in younger patients. Thus, samples taken for histological clarification should also include samples for TB diagnosis (ZN stain, PCR, culture).

Germany has a low incidence of TB; in 20155865 cases were reported, an increase of $29 \%$ over the prior year. This increase is almost exclusively due to emigration. In 2014 the portion of patients not born in Germany was $64 \%$. The appearance of TB infection among emigrants frequently appears in the course of the first few years after arrival. It is thus not surprising that in 2014 only 409 cases ( $10.9 \%$ ) were identified during the intake examination. The persistent fear of TB transmission of emigrants from high prevalence regions to the autochthonous low prevalence region has still not been confirmed [11 - 14].

Postprimary pulmonary TB typical is manifested as consolidating pneumonia with cavitations $[15,16]$, which can be absent in the early exudative phase, and are found in only about $50 \%$ of cases. Specific cavitations arise from encroachment of caseous necrosis on the bronchial system; they are thick-walled with irregular contours. Usually postprimary pulmonary TB is localized in the apical and posterior upper lobe segments and in the apical lower lobe segments. There is frequently endobronchial pathogen spread via the cavitations encroaching on the bronchial system which in the $\mathrm{CT}$ appears as bronchiolitis with sharply delineated centrilobular nodules combined with centrilobular branching lines ("tree-in-bud" pattern). In about $5 \%$ of postprimary pulmonary TB cases, tuberculomas rather than infiltrates represent the radiological finding. Tuberculomas are sharply-defined focal masses with diameters between 0.5 and $4 \mathrm{~cm}$. They originate as granulomas formed from the residue of the primary complex, frequently exhibit calcifications and can demonstrate satellite foci in their vicinity [17]. An intact cellular immune response is required for the formation of the typical signs of postprimary pulmonary TB.

Since the primary TB infection (primary complex) leads to hematogenic spread in principle, postprimary TB can develop in every organ. There is subclinical primary infection and spread in 


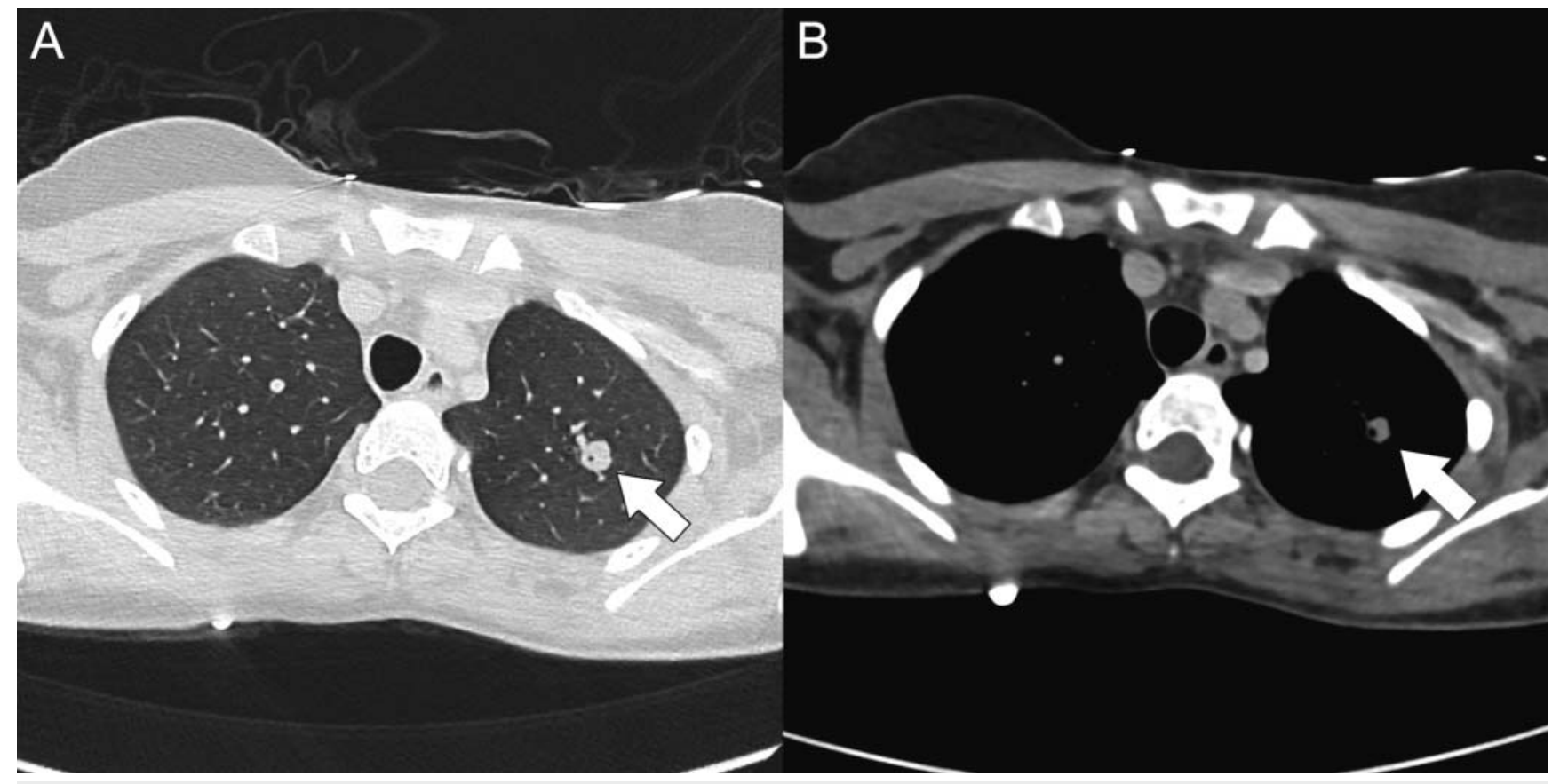

- Fig. 4 Patient with postprimary lung tuberculosis. CT shows a well-defined solid nodule in the left upper lobe with punctuate intralesional gas indicating beginning cavitation of the tuberculoma (arrows in $\mathbf{A}$ and $\mathbf{B}$ ).

approx. $90 \%$ of infected patients. The radiological appearance of extrapulmonary postprimary TB is non-specific and particularly mimics tumor diseases [18]. Signs of active or residual pulmonary TB are present in fewer than $50 \%$ of conventional radiological examinations, thus, extrapulmonary TB cannot be ruled out due to the absence of signs of primary lung infection (pleural scarring calcifications).

In descending frequency, extrapulmonary tuberculosis affects lymph nodes, pleura, urogenital tract, bones and/or joints, abdominal cavity and/or gastrointestinal tract and the CNS. In this regard, refer to the relevant preparatory studies, e. g., by Heye et al. [18]. Imaging of peritoneal TB is hardly distinguishable from peritoneal carcinosis. There is a diffuse or nodular thickening of the parietal and visceral peritoneum and the greater omentum with ascites. Web-like structures in ascites, lymphadenopathy with necrosis and the absence of a primary malignancy are indicative of tubercular peritonitis. Peritoneal TB can involve intestinal TB. Most frequently this affects the ileocecal region and appears as circular wall thickening. Formation of fistulas and fibrous stenosis is possible. The visual impression of intestinal TB resembles that of Crohn's disease.

\section{Case History: Postprimary Pulmonary TB}

17-year-old female patient from Eritrea, living in Germany since May 2014. Initial hemoptysis in February 2014. CT disclosed thoracic mass in left upper lobe of lung. Patient was hospitalized in June 2014 to rule out tuberculosis. Bronchial lavage was negative for tuberculosis. In December 2014 patient underwent CT-guided puncture sample of pulmonary nodule. There was a finding of histopathological granulomatous necrotizing disease. Further histopathological examinations did not indicate TB. In April 2015 patient was again hospitalized for hemoptysis. Sputum culture revealed positive evidence of tuberculosis.

CT in the interim revealed a sharply-defined nodule in the apex of the left lung ( $\triangleright$ Fig. 4). A small gas inclusion was located in the lesion. This was a tuberculoma with beginning necrosis.

\section{Case History: Postprimary TB of the Gastrointestinal Tract}

29-year-old female patient living in Europe for 18 months. Patient experienced increasing waist circumference for 3 months, night sweats, $10 \mathrm{~kg}$ weight loss in past 6 months and fatigue. Sonography exhibited ascites. Abdominal MRI demonstrated diffuse peritoneal thickening affecting the greater omentum, small intestine mesentery and parietal peritoneum ( $\mathbf{F i g . 5}$ ). There was no lymph node enlargement. Laparoscopic peritoneal biopsy was performed for an histopathological examination and to discover mycobacteria. There was evidence of epithelioid cell granuloma, mycobacterium tuberculosis complex PCR positive.

\section{KEY POINTS}

- In addition to pneumonia with/without cavitation, postprimary pulmonary TB can also manifest as developing granuloma.

- Under immunosuppression the radiological manifestation of pulmonary TB is frequently atypical.

- In patients who have emigrated, extrapulmonary TB is an important differential diagnosis to malignant diseases. 


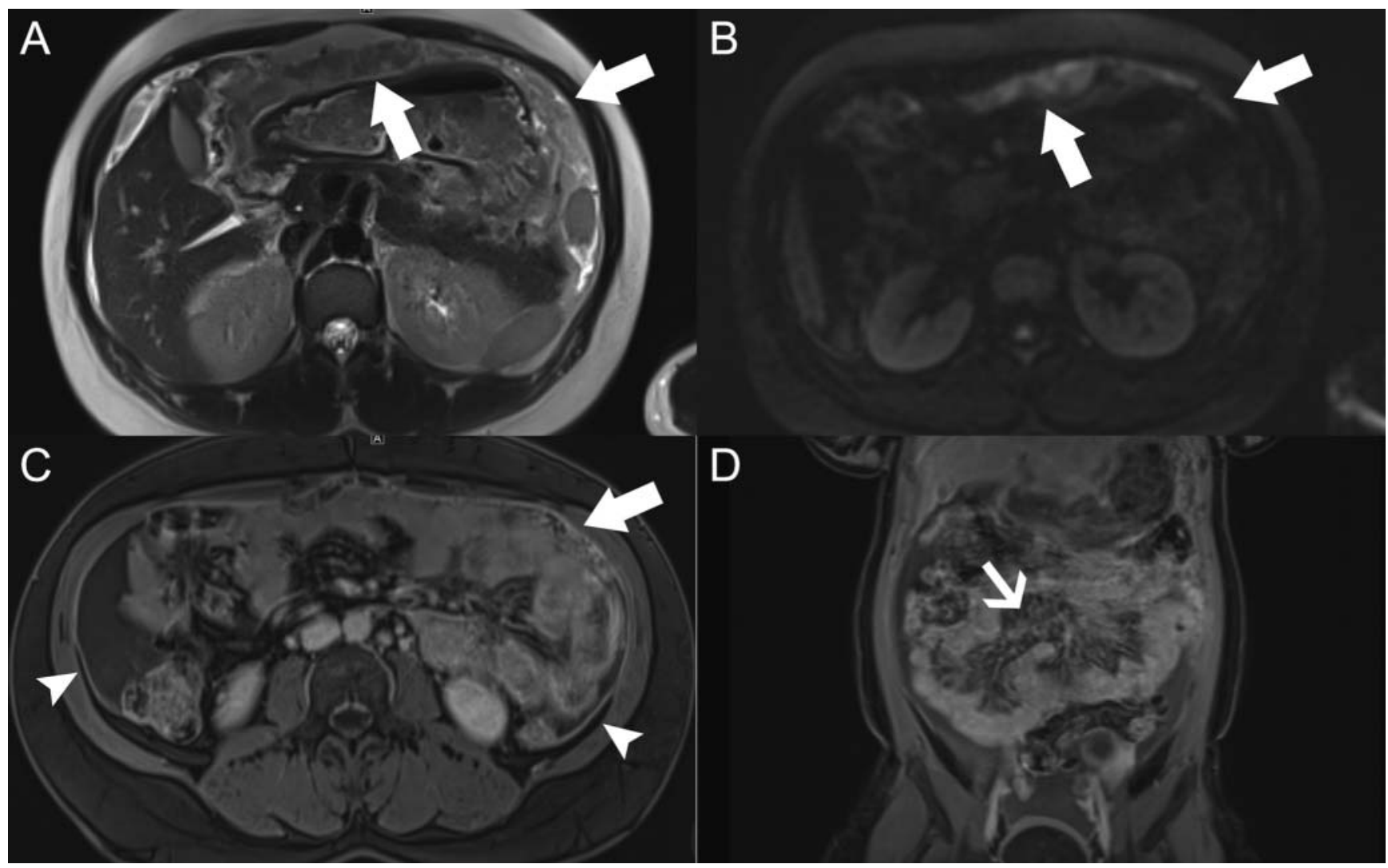

- Fig. 5 Patient with peritoneal tuberculosis. MRI in T2-weighting and diffusion weighting shows a plaque-like infiltration of the greater omentum (arrows in $\mathbf{A}$ and $\mathbf{B}$ ). Post contrast there is marked thickening of the parietal peritoneum (arrow heads in $\mathbf{C}$ ) and the small intestine mesentery (arrow in D) apart from omental infiltration (arrow in C).

\section{Schistosomiasis}

Infection by schistosomes is a chronic infectious disease caused by fresh water contact with the parasite. The disease is wide-spread in Sub-Saharan Africa as well as Southeast Asia and South America. Radiologically detectable end organ complication rate is 10 $20 \%$ of cases. An immune response to schistosomes is the source of systemic and organ-specific pathology. Diagnosis is based on evidence of worm eggs in urine or stools, both histopathologically and serologically $[19,20]$.

Hepatosplenic schistosomiasis is caused by S. mansoni in the catchment of our patients (Sub-Saharan Africa). Periportal liver fibrosis is the primary pathology of the chronic infection. Adult $S$. mansoni live in the venules of the gastrointestinal tract. Via the portal vein system the eggs embolize into the periportal fields of the liver where granulomas are induced resulting in liver fibrosis. There is an increased risk of hepatocellular carcinoma. The infection can result in wall thickness, polyps and strictures of the gastrointestinal tract.

In sonography, periportal liver fibrosis appears as hyperechogenic thickening of the walls of the portal vein. CT exhibits native hypodense band-like structures along the portal vein system. Fibrosis strands are hyperintense in T2-weighted MRI. After contrast administration, CT and MRI venous series show increased accumulation in the periportal fibrosis [21].
Following the guidelines of Richter et al. there is a distinction between central and peripheral fibrosis which typically appear in combination ( $\triangleright$ Fig. 6) [22]. The fibrosis patterns reflect the severity of liver damage. Infection by S. mansoni is dominated by central periportal fibrosis which spreads hilifugally into the periphery. A "turtle back" appearance is a late effect. Penetration of the hepatic surface results in fibrous septation along the periportal fields reaching to the hepatic capsule. "Starry sky pattern" (pattern B) and "pipe stem pattern" (pattern C) with emphasized hyperechogenic reflections in the sonogram along the very small and small branches of the portal vein are early manifestations of peripheral fibrosis, which only in combination with central periportal fibrosis indicates the likelihood of schistosomiasis caused by S. mansoni [22].

The urogenital form of schistosomiasis is primarily caused by $S$. hematobium dwelling in the venules of the urinary tract. The bladder is usually affected; schistosomiasis causes hypertrophy of the bladder mucosa and thickening of the bladder wall to more than $5 \mathrm{~mm}$. Focal wall irregularities with formation of pseudopolyps and masses are also found. Egg-shaped calcification of the bladder wall is pathognomonic of schistosomiasis of the bladder. The diseases results in obstructive uropathy. Granulomatous inflammation in the upper urinary tract leads to fibrosis along the ureters and finally ureter dilation, hydronephrosis and renal failure. The risk of developing bladder cancer is increased. 


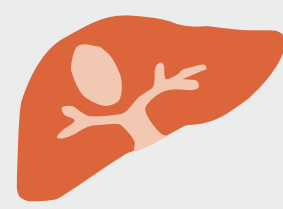

a: Normal

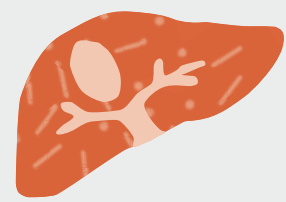

c: Peripheral fibrosis

Pipe stem pattern

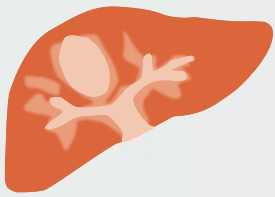

e: Central fibrosis

Widening of central periportal space and fibrosis

spots in the parenchyma

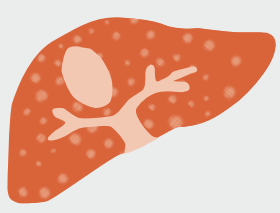

b: Peripheral fibrosis Starry sky pattern

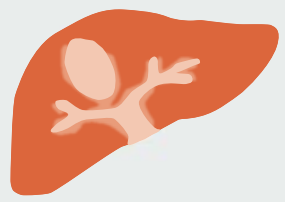

d: Central fibrosis Widening central periportal space

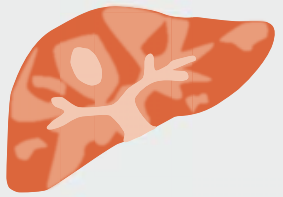

f: Central fibrosis

Fibrosis filaments up to liver capsule

- Fig. 6 Patterns of periportal liver fibrosis in schistosomiasis. Patterns $b$ and $b$ alone are not evidentiary of periportal fibrosis and may be observed in the healthy population as well. Central periportal fibrosis patterns (d, e, and $\mathbf{f}$ ) may occur in combination with peripheral fibrosis.

\section{Case History: Liver Fibrosis resulting from S. Mansoni Infection}

25-year-old male patient from Eritrea, otherwise without complaints, presented with pancytopenia discovered as an incidental finding. Sonography exhibited a centrally dominant liver fibrosis and splenomegaly ( $\triangleright$ Fig. 7). According to the guidelines of Richter et al. there was a combination of fibrosis patterns $D$ and $C$. Esophageal varices II-III were gastroscopically detectable. Serology and evidence of S. mansoni eggs in the stool were positive.

\section{KEY POINTS}

- Depending on the pathogen, chronic schistosomiasis especially causes damage to the liver and bladder.

- In the emigration context, schistosomiasis should be included in the differential diagnosis of patients with liver parenchyma damage and portal hypertension.

- Periportal fibrosis in schistosomiasis is distinct from cirrhosis of the liver.

\section{Visceral Leishmaniasis}

Visceral leishmaniasis (VL) is most likely to be confused with hemato-oncological diseases. Radiologically, pronounced hepatosplenomegaly and intra-abdominal lymph node enlargement may be noticed in patients. Patients originating from a high-prevalence region for $V L$ and presenting with fever, splenomegaly, and pancytopenia should be checked for VL.

$\mathrm{VL}$ is a slowly progressive disease with incubation periods ranging from 10 days to 2 years. Longer incubation periods are especially possible under immunosuppressive therapy or with HIV comorbidity. Untreated, symptomatic VL is usually fatal. The diagnosis can be made by detection of antibodies and amastigote parasite forms in the bone marrow. Long-term follow-up due to recurrence is necessary after therapy, especially for immunosuppressed patients.

Radiological signs of VL are non-specific. Sonographic findings include splenomegaly and hepatomegaly. Lymphadenopathy can be observed. The presence of multiple nodular masses in the liver and spleen has been reported, but the parenchyma is usually homogeneous $[23,24]$. Spleen lesions are hypoechogenic in the sonogram, hypointense in the T2-weighted MRI and hypointense in the CT compared to the other parenchyma $[19,20]$.

\section{Case History: VL}

25-year-old male patient from Eritrea, living in Germany only a few days. He presented due to left-side stomach ache and frequent nose bleeds. The physical examination revealed cachexia, hepatitis and splenomegaly. Laboratory results showed pancytopenia Leishmania serology was positive. Imaging revealed splenomegaly with focal splenic lesions ( $\triangleright$ Fig. 8). Under specific therapy the patient showed clinical improvement with weight gain and reduced anemia.

\section{KEY POINTS}

- VL is an important differential diagnosis to hemato-oncological diseases.

- Hepatosplenomegaly with or without focal lesions is a nonspecific main finding.

\section{Cysticercosis}

Cysticercosis is a disease caused by the fecal-oral ingestion of the eggs of the pork tapeworm, with high prevalence in West, Central and South Africa, Southeast Asia as well as in Central and South America. The cysticerca can be found in the brain parenchyma, subarachnoidally, intraventricularly or in the spinal cord, as well as in the skeletal and cardiac muscles and other tissues [25, 26].

Neurocysticercosis is one of the world's leading causes of epilepsy. Seizures are the most common clinical presentation followed by focal neurological signs and hydrocephalus. The diagnosis is radiological, serology complements imaging and has sensitivity problems. The therapy decision is based on the number and location of cysts (extra- or intraparenchymal cysts) and the 


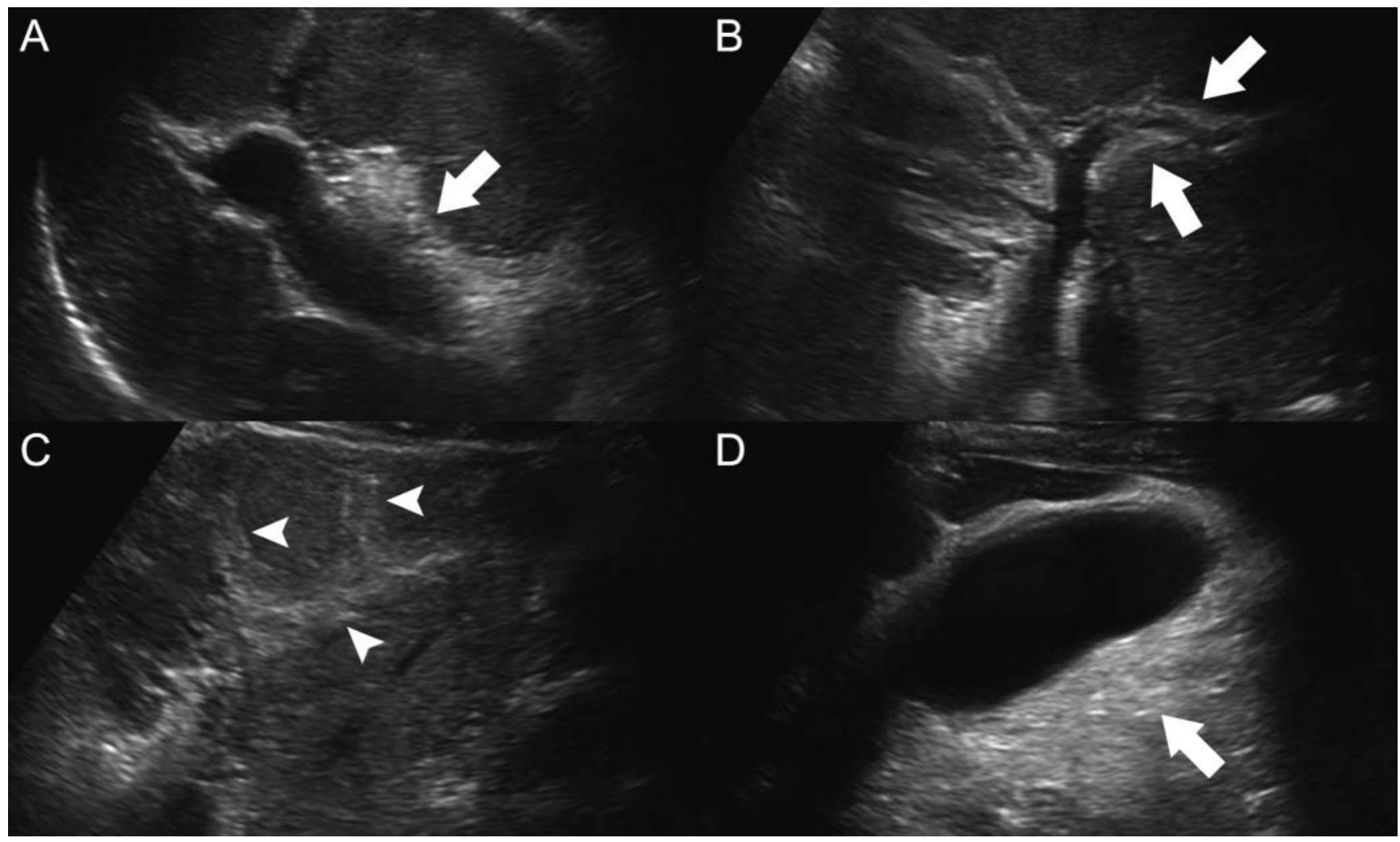

- Fig.7 Patient with periportal liver fibrosis due to chronic infection with Schistosoma mansoni. Ultrasound shows hyperechoic fibrotic bands that extend from the central periportal space (A) along segmental and subsegmental portal vein branches (B and $\mathbf{C})$. The gall bladder is surrounded by fibrotic tissue as well (D). According to Richter et al. a combination of fibrosis patterns $\mathrm{d}$ and $\mathrm{c}$ is present.

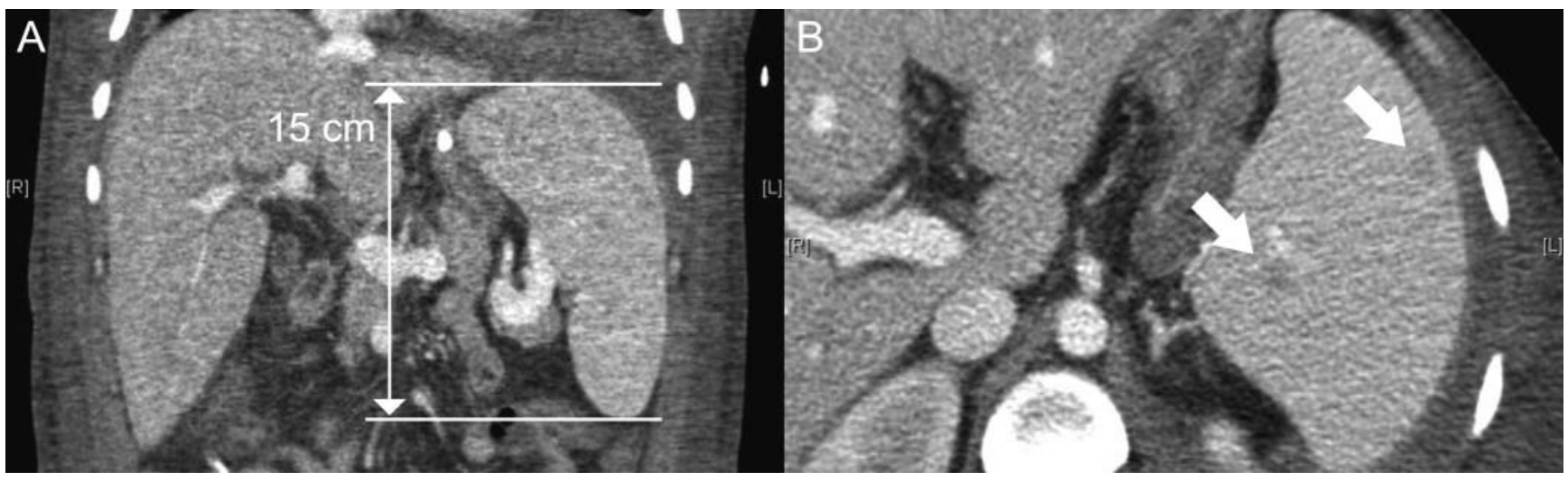

- Fig. 8 Patient with visceral leishmaniosis. Contrast-enhanced CT shows unspecific splenomegaly (A). There are several small hypodense splenic nodules (B).

radiological determination of cyst viability. The therapeutic spectrum ranges from symptomatic antiepileptic and/or anti-edematous therapy to a specific antiparasitic treatment individually tailored based on the above-mentioned criteria.

Based on imaging, neurocysticercosis is divided into 4 stages, which reflect the development of the parasite and the host response [27]: vesicular stage, colloidal vesicular stage, granulomatous-nodular stage and calcified nodular stage. Initially neurocysticercosis is not revealed in imaging. In the vesicular stage, a fluid-fil- led cyst has developed which measures up to $20 \mathrm{~mm}$. In the interior the scolex (head of the larva) can often be distinguished. The scolex has a diameter of 2 to $4 \mathrm{~mm}$ and appears bright compared to the cystic fluid in T1 weighted MRI and CT. The cyst wall is thin and there is no perifocal edema. The cyst begins to degenerate in the colloidal vesicular stage. An inflammatory host response occurs, inducing the formation of a fibrous capsule which can cause local edema. The extent of the immune response is variable and can result in diffuse encephalitis. At this time MR or CT images reveal 


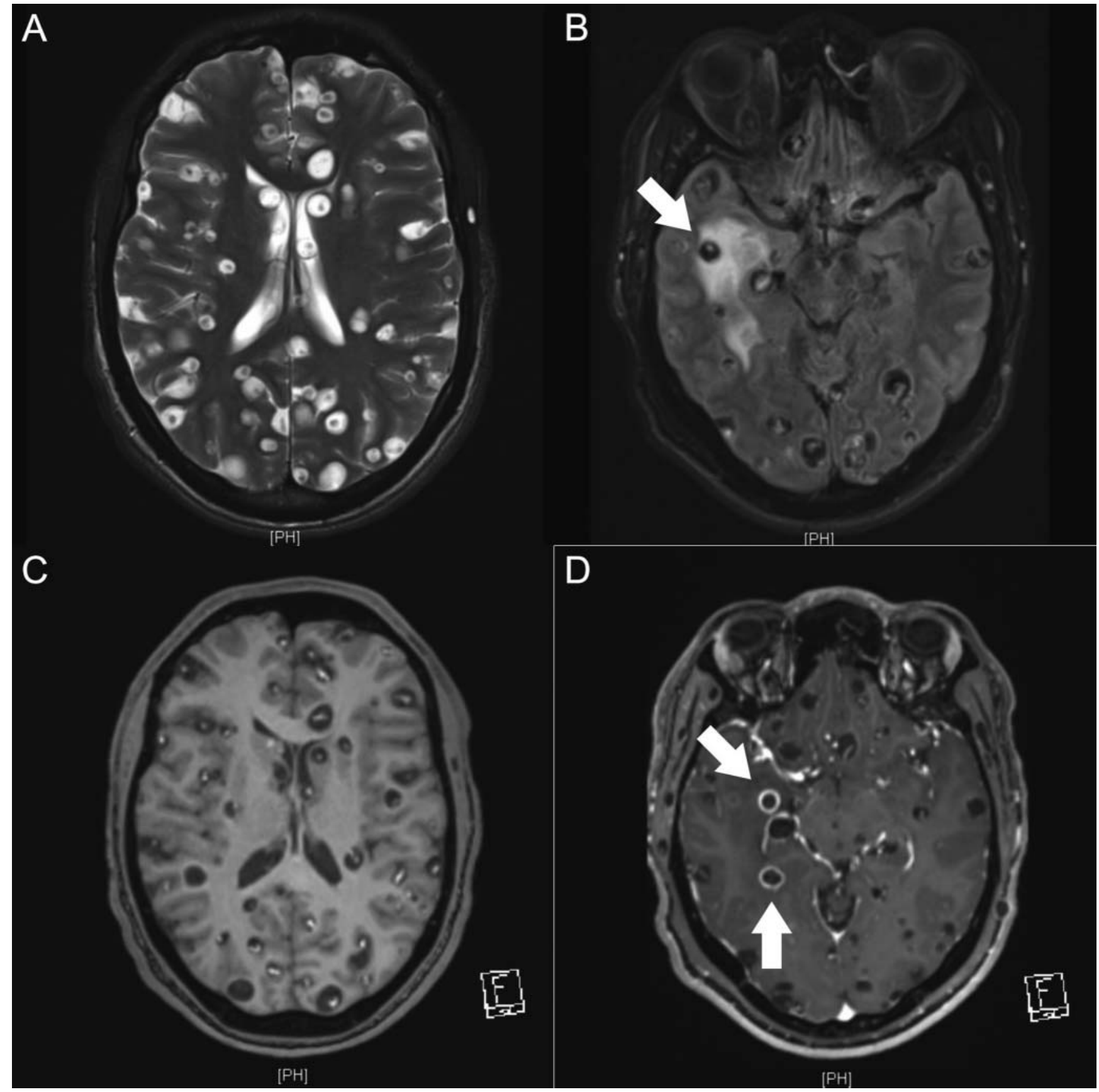

- Fig. 9 Patient with neurocysticercosis. Cranial MRI shows multiple cysticerci in the vesicular stage (A, T2-weighting). In the right temporal lobe there is perifocal edema indicating a colloidal vesicular stage with inflammatory host reaction (B, FLAIR). The pre-contrast T1-weighting shows the scolices as intralesional hyperintense nodules (C). Post contrast cysts in the colloidal vesicular stage demonstrate ring enhancement (D).

ring-shaped contrast-enhanced lesions with or without local edema. The cyst content in the T1-weighted MRI may show a signal increase which is explained by debris in the cystic fluid. In the granulomatous-nodular stage the cyst contracts, resulting in a solid nodule that enhances post-contrast and is surrounded by slight perifocal edema. In the calcified nodular stage the granuloma is fully calcified and the lesion is inactive. In the MRI, these lesions are correspondingly hypointense in T1- and T2-weighted images.
Occasionally, perifocal edema associated with episodic seizures can also be observed in the calcified nodular stage [28].

\section{Case History: Cysticercosis}

22-year-old male patient from Togo with multiple pulmonary focal masses discovered at screening chest x-ray. In addition, radiological examinations revealed multiple cystic masses in the 
- Table 1 Worldwide cases of individual cases of infectious diseases in 2010, adapted from [29, 30] and high-prevalence regions.

\begin{tabular}{|c|c|c|}
\hline disease & number of cases & high-prevalence regions \\
\hline schistosomiasis & 252 million & $\begin{array}{l}85 \% \text { of infections in Sub-Saharan Africa, less common in Middle East, South } \\
\text { America, Southeast Asia }\end{array}$ \\
\hline cysticercosis & 1.4 million & central and South America, Sub-Saharan Africa, Southern Asia and China \\
\hline cystic echinococcosis & 1.1 million & $\begin{array}{l}\text { middle and Near East, North and East Africa, former Soviet Union, China, } \\
\text { Mongolia, South America }\end{array}$ \\
\hline visceral leishmaniasis & 76000 & east Africa, India, Mediterranean region, Middle East, Central and South America \\
\hline tuberculosis & $\begin{array}{l}10.4 \text { million new TB cases } \\
\text { in } 2015\end{array}$ & sub-Saharan Africa, Asia http://www.stoptb.org/countries/tbdata.asp \\
\hline
\end{tabular}

musculature. The patient's history included epilepsy. MRI of the skull exhibited numerous masses without perifocal edema. Twelve months after the diagnosis, the patient increasingly experienced headaches. A further MRI showed new perifocal edema surrounding some lesions ( $\mathbf{F i g . 9}$ ). Cysticercosis-related masses were found in the vesicular and colloid-vesicular stage. Antiparasitic therapy was not pursued due to the risk of cerebral edema caused by the decay of the parasitic cysts. The patient was treated with intermittent steroid therapy.

\section{KEY POINTS}

- Neurocysticercosis is a significant cause of epilepsy.

- The development of cysticerca is radiologically observable and is the basis for classification into stages, which in turn, determines the therapy.

- The spontaneous and, in particular, drug-induced degradation of the cysts causes immunological host reactions, negatively affects interpreting the images and clinical signs, and can be life-threatening due to the development of cerebral edema.

\section{Discussion}

Radiologists cannot be expected to be aware of the global geography of disease, even though imported diseases that are rare among us are among the most common in other areas of the world ( $\triangleright$ Table 1) $[29,30]$. Although this article focuses on infectious diseases, a limited selection can contribute to an understanding of significant emigration-related health issues.

Radiology plays a decisive role in primary diagnosis as well as in staging of the diseases presented in the case series. Clinically, CE and neurocysticercosis are unspecific, and serological testing is not reliable. For both of these diseases, radiology offers highly sensitive and specific examination techniques. Radiologically, CE, schistosomiasis and neurocysticercosis are classified according to stages. Therapeutic decisions and follow-up examinations are based on such classifications. Therapy success is defined by stage progression in CE and neurocysticercosis.
Expectations are crucial for the differential diagnosis of a finding. This determines the order in which differential diagnoses are processed in the course of the patient's examination. However, this is fundamentally different in patients with differing disease epidemiologies. Thus, the probability of a malignancy versus TB in patients of the same age group from different regions of the world can vary substantially. While a mass in the right upper lobe of the lung in a middle-aged patient in Germany presenting with weight loss, night sweats and fever might be suspected of a malignancy, TB might be on the list of differential diagnoses of a patient from Pakistan.

Among the diseases discussed, only open pulmonary tuberculosis is contagious for health care workers and fellow patients. On the general population level, there is currently no measurable transfer of TB from emigrants from high-prevalence TB areas to the native population. In the health care sector, due to the close contact of care personnel and co-patients with potentially infectious diseases such as open pulmonary tuberculosis, a high level of caution and protective measures should be observed to safely rule out the risk of infection. Such measures include oral-nasal protection on the part of the patient and FFP-2 respiratory masks for hospital personnel and visitors.

In conclusion, a simple but very effective rule: the differential diagnosis in patients from other continents should include the possibility of uncommon imported diseases. When in doubt, seek advice. Tropical medical centers specializing in infectious diseases are a good resource for radiological collaboration.

Correction: Stojkovic M, Müller J, Junghanss T et al. Radiological Diagnoses in the Context of Emigration: Infectious diseases. Fortschr Röntgenstr 2017; DOI: 10.1055/s-0043-120764

A corrected version of the above mentioned article was published at December 19th 2017. The name of the first author has been corrected to Marija Stojkovic.

\section{Conflict of Interest}

The authors declare that they have no conflict of interest. 
Literature

[1] Wilson S. Infectious diseases of specific relevance to newly arrived migrants in the EU EEA; 2015: 1 -6

[2] International Air Transport Association. Airlines to Welcome 3.6 Billion Passengers in 2016. http://www.iata.org/pressroom/pr/Pages/2012-1206-01.aspx, Zugriff am 14.10.2017

[3] Bundesamt für Migration und Flüchtlinge. Das Bundesamt in Zahlen Asyl. Migration und Integration 2016

[4] Brunetti E, Kern P, Vuitton DA et al. Expert consensus for the diagnosis and treatment of cystic and alveolar echinococcosis in humans. Acta Trop 2010; 114: 1 - 16

[5] Stojkovic M, Rosenberger K, Kauczor H-U et al. Diagnosing and Staging of Cystic Echinococcosis: How Do CT and MRI Perform in Comparison to Ultrasound? PLoS Negl Trop Dis 2012; 6: e1880 -e1888

[6] Junghanss T, da Silva AM, Horton J et al. Clinical management of cystic echinococcosis: state of the art, problems, and perspectives. Am J Trop Med Hyg 2008; 79: 301-311

[7] Hosch W, Stojkovic M, Janisch T et al. The role of calcification for staging cystic echinococcosis (CE). Eur Radiol 2007; 17: 2538-2545

[8] Saksouk FA, Fahl MH, Rizk GK. Computed tomography of pulmonary hydatid disease. J Comput Assist Tomogr 1986; 10: 226-232

[9] Koul PA, Koul AN, Wahid A et al. CT in Pulmonary Hydatid Disease. Chest 2000; 118: 1645-1647

[10] Singh U, Kumar S, Gour H et al. Complicated hydatid cyst and "air bubble" sign: a stepping-stone to correct diagnosis. Am J Case Rep 2015; 16: $20-24$

[11] Barniol J, Niemann S, Louis VR et al. Transmission dynamics of pulmonary tuberculosis between autochthonous and immigrant sub-populations. BMC Infect Dis 2009; 9: 197

[12] Dahle UR, Eldholm V, Winje BA et al. Impact of immigration on the molecular epidemiology of Mycobacterium tuberculosis in a low-incidence country. Am J Respir Crit Care Med 2007; 176: 930 - 935

[13] Verver S, van Soolingen D, Borgdorff MW. Effect of screening of immigrants on tuberculosis transmission. Int J Tuberc Lung Dis 2002; 6: $121-129$

[14] Davies P. Clinical tuberculosis: a practical handbook. Boca Raton: CRC Press; 2016

[15] Leung AN. Pulmonary tuberculosis: the essentials. Radiology 1999; 210 : $307-322$
[16] Eisenhuber E, Mostbeck G, Bankier A et al. Radiologic diagnosis of lung tuberculosis. Radiologe 2007; 47: 393-400

[17] Beigelman C, Sellami D, Brauner M. CT of parenchymal and bronchial tuberculosis. Eur Radiol 2000; 10: 699-709

[18] Heye T, Stoijkovic M, Kauczor HU et al. Extrapulmonary tuberculosis: radiological imaging of an almost forgotten transformation artist. Rofo 2011; 183: 1019-1029

[19] Gibson GM, Arnold C, Ravi KumarAS. 18F-FDG uptake in multiple splenic foci on PET/CT: an unusual case of visceral leishmaniasis. Clin Nucl Med 2014; 39: $828-830$

[20] Yapar AF, Reyhan M, Kocer NE et al. Diffuse splenic F-18 FDG uptake in visceral leishmaniasis. Clin Nucl Med 2011; 36: 1041 - 1043

[21] Manzella A, Ohtomo K, Monzawa S et al. Schistosomiasis of the liver. Abdom Imaging 2008; 33: 144-150

[22] Richter J, Hatz C, Campagne G et al. Ultrasound in Schistosomiasis. World Health Organization 2000; Ultrasound in schistosomiasis: a practical guide to the standard use of ultrasonography for assessment of schistosomiasis-related morbidity.

[23] Bükte Y, Nazaroglu H, Mete A et al. Visceral leishmaniasis with multiple nodular lesions of the liver and spleen: $\mathrm{CT}$ and sonographic findings. Abdominal Imaging 2004; 29: 82-84

[24] Raeymaeckers S, Docx M, Demeyere N. MRI-findings of nodular lesions in an enlarged spleen, associated with visceral Leishmaniasis. European Journal of Radiology 2012; 81: 2550-2553

[25] Garcia HH, Nash TE, Del Brutto OH. Clinical symptoms, diagnosis, and treatment of neurocysticercosis. The Lancet Neurology 2014; 13: 1202 1215

[26] Del Brutto OH, Nash TE, White AC et al. Revised diagnostic criteria for neurocysticercosis. J Neurol Sci 2017; 372: 202-210

[27] Noujaim SE, Rossi MD, Rao SK et al. CT and MR imaging of neurocysticercosis. Am J Roentgenol 1999; 173: 1485-1490

[28] Nash TE, Pretell E], Lescano AG et al. Perilesional brain oedema and seizure activity in patients with calcified neurocysticercosis: a prospective cohort and nested case-control study. The Lancet Neurology 2008; 7: 1099-1105

[29] Global tuberculosis report 2016. Who 2016. http://www.who.int/tb/ publications/global_report/en/, Zuletzt zugegriffen am 15.10.2017

[30] Hotez P], Alvarado M, Basáñez M-G et al. The global burden of disease study 2010: interpretation and implications for the neglected tropical diseases. PLoS Negl Trop Dis 2014; 8: e2865 
